\section{Geografías menores, geografías cotidianas: la construcción del ciudadano nacional en Chile Chico, Región de Aysén ${ }^{*}$}

\section{Minor geographies, everyday geographies: the construction of the national citizen in Chile Chico, Region of Aysén}

\author{
ANDRÉS NúÑEZ $Z^{*}$ \\ Matthew C. Benwell \\ ENRIQUe ALISTE \\ JAVIERA MUÑOZ
}

\section{Resumen}

Planteamos que el ciudadano nacional no solo se produce desde el centro político, desde el orden discursivo de índole nacional sino, especialmente, desde su devenir cotidiano, desde su quehacer silencioso y menor que implican sus prácticas, donde ellos co-fabrican sentidos nacionales desde los simbolismos y materialidades presentes en sus respectivos territorios. Mecanismos locales que dialogan con la escala nacional como la educación, el espacio doméstico (hogar) y espacio público, banderas, murales y ceremonias se despliegan mediados por sus propias trayectorias culturales. Tomando el caso de Chile Chico (Aysén, Chile), que se encuentra a solo 14 kilómetros de Los Antiguos (Santa Cruz, Argentina) constatamos que allí se desenvuelve un habitar fronterizo que proyecta rasgos tanto de índole nacionalista como otros que rebasan lo nacional para llegar a comprenderse como un espacio de memoria común. Así, los ciudadanos, desde sus geografías menores y cotidianas, se tornan tanto agentes geopolíticos que producen y rehacen el sentido comunitario y de pertenencia a la nación como, a su vez, en agentes de una memoria que pone en valor un devenir que les pertenecía incluso antes que llegará la nación y sus límites.

Palabras clave: Geografías menores, nacionalismo banal, ciudadano nacional, frontera.

Los autores agradecen al proyecto Fondecyt $N^{\circ} 1170643$, que hizo posible la investigación y trabajo de campo. A su vez, agradecen el relevante apoyo prestado en esta investigación por Valko Durán y Tania Vas, ambos chilechiquenses.

** Instituto de Geografía, Pontificia Universidad Católica de Chile (Chile). E-mail: aanunezg@uc.cl

*** School of Geography, Politics and Sociology, Newcastle University (Reino Unido). E-mail: matthew.benwell@newcastle.ac.uk

**** Departamento de Geografía, Universidad de Chile. E-mail: ealiste@uchilefau.cl

** Instituto de Geografía, Pontificia Universidad Católica de Chile (Chile). E-mail: jmunoz10@uc.cl 


\section{Abstract}

In the following paper we develop the idea that the national citizen is not only formed from the political center of the nation. The concept of the nation is produced and reproduced through daily practices of the everyday, through citizens' engagements with the symbols and materialities of their surrounding territories. The local and the national are brought into dialogue in the context of educational, domestic (home) and public spaces, where things like flags, street art and commemorative ceremonies are engaged through their own cultural trajectories. Citizens living in the border town of Chile Chico (Aysén, Chile), which is only 14 kilometers away from Los Antiguos (Santa Cruz, Argentina), identify with aspects of the nation, as well as sharing spaces of common memory that go beyond the national level. Through these acts, citizens, in their minor and daily geographies, become both geopolitical agents that produce and reproduce a sense of belonging to the nation, as well as agents whose memory has given value to their own forthcoming, even before the nation and its borders had arrived.

Key words: Minor geographies, banal nationalism, national citizens, borders

\section{Introducción}

La nación puede ser entendida como una producción del mundo moderno que responde a la necesidad de generar una identidad común, que actúa, en consecuencia, como marco organizador de un vasto territorio con habitantes que compartirían características relativamente similares a los que es posible llamar ciudadanos nacionales. Esta forma de organización territorial potenciada por los Estados desde una escala mayor a la del habitante simple, responde a un proceso intencionado y normalizado a través del tiempo, buscando mostrar una imagen estática y rígida que otorgue la homogeneidad indispensable para generar la noción de "comunidad". Desde esta perspectiva, la nación, y por ende el nacionalismo, es comprensible como artefacto cultural o producto cultural donde uno de sus resultados sería la adherencia del grupo a dicha comunidad o sentido común (Anderson 1983). Uno de los aspectos más relevantes de este nivel escalar es que busca tanto afianzar un modelo hegemónico de organización territorial así como modos y estrategias de cohesión social.

Lo expuesto con antelación, sin embargo, puede también ser observado desde una escala menor, buscando comprender al nacionalismo como las prácticas que expresan aquel sentimiento nacional comunitario y aunque ligado a él también lo mediarían desde la cotidianidad y su habitar existencial (Núñez et al. 2017). En lo cotidiano es posible reconocer distintos discursos, prácticas e incluso trayectorias y movilidades que devienen de lo nacional; a esto se le ha llamado Nacionalismo Banal (Billig, 1995) y es uno de los conceptos que estructura esta investigación.

Desde una escala local, desde el lugar, es posible identificar una dinámica que contrasta con el sentido rígido y permanente de la nación. Se reconoce la producción del ciudadano nacional a través de diferentes impulsos dirigidos desde discursos dominantes, pero también desde experiencias cotidianas que generan una atmósfera que está en constante desarrollo y movimiento, en constante producción. En otras palabras, "hace falta analizar la manipulación 
por parte de los practicantes que no son sus fabricantes. Solamente entonces se puede apreciar la diferencia o la similitud entre la producción de la imagen y la producción secundaria que se esconde detrás de los procesos de su utilización" (De Certeau 2010:43).

El interés de esta propuesta se centra en ese nacionalismo que surge desde "abajo", desde las prácticas, en la reproducción de la nación desde una geografía menor (Oliveira Jr. 2009). Nos interesa identificar desde esta escala menor los dispositivos y elementos propulsores de la producción del ciudadano nacional en Chile Chico, ciudad ubicada en la Región de Aysén, en la ribera del Lago General Carrera y limítrofe con su par argentino, Los Antiguos. Esta relación de vecindad, que desde la escala nacional comunitaria supone la existencia de otro distinto y ajeno, es decir, otra nación, en la cotidianidad las imbricaciones sociales dialogan con y desde la cercanía entre ambos poblados (14 kilómetros) así como desde una memoria común desde sus inicios, además de los flujos constantes de un lado a otro (Ver Figura 1)

En efecto, en el área de estudio, el Complejo Fronterizo Jeinimeni es el elemento más evidente de lo que representa el concepto de nación, pudiendo ser entendido como un elemento rígido de presencia estatal. Sin embargo, las relaciones existentes en el contexto del vínculo entre Chile Chico y Los Antiguos, que se abordan en este artículo, llevan a atender la construcción del ciudadano nacional desde una escala mínima, desmitificando el concepto de nacionalismo como un elemento exclusivo de las naciones y de los Estados, mostrándolo como un producto de las relaciones dinámicas de una frontera permeable. De esta manera, surgen interrogantes sobre cómo se construye el ciudadano nacional desde la experiencia de habitar en una zona interfronteriza, cómo se presenta el rol del Estado y qué alcances tiene en lo cotidiano.

Figura 1. Contexto del área de estudio

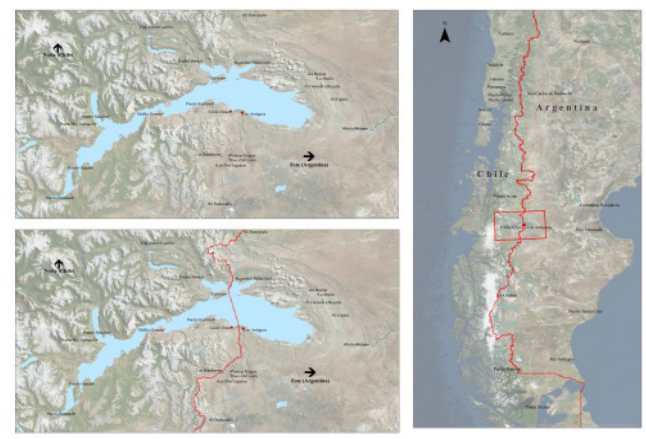

Fuente: Los autores

Interesa también desmitificar el concepto de nacionalismo como algo ligado a la óptica y geopolítica de los estado-nación, es decir, solo como dispositivo colectivo de enunciación. Al hablar sobre lo banal del nacionalismo nos referimos a lo característico de lo cotidiano, esto es, lo presente en el día a día, aunque no como un nacionalismo sin importancia o vulgar. El concepto de banal se entiende desde las prácticas y objetivos que desde una esfera estatal permea, es mediado y se re-produce en y desde lo cotidiano, en el habitar existencial, en la experiencia, en la rutina y en el diario vivir.

Desde un punto de vista metodológico, para la realización de esta investigación se indagó en terreno entre mayo y agosto de 2018, logrando pesquisar información desde diferentes fuentes locales de Chile Chico a través de entrevistas semi estructuradas. Sin embargo, los focus 
group realizados con jóvenes estudiantes del Liceo Luisa Rabanal Palma en Chile Chico se presentan como una instancia fundamental para dibujar la imagen actual del ciudadano nacional, permitiendo reconocer el actuar de la memoria. Se logra así demostrar los matices que puede alcanzar el concepto de nacionalismo al proponer el estudio desde una escala mínima, lo que no estuvo exento de dificultades. Desde el trabajo de campo se hace muy complejo abordar el estudio del nacionalismo sin una previa deconstrucción, ya que, como se ha mencionado, el carácter implícito y omnipresente del sentimiento nacional se encuentra interno en cada individuo, incluso en los investigadores, por lo que fue necesario llevar a cabo un largo proceso de observación e implicación en el área de estudio.

En definitiva, desde este punto de vista, es relevante resaltar que el nacionalismo dominante y el nacionalismo banal se relacionan y se vinculan desde lenguajes que desenvuelven procesos de hibridez cultural tanto desde lo discursivo como en el ámbito de sus prácticas. Allí, la identidad nacional se relaciona con la escala de la nación homogénea, pero a su vez con una de tipo regional-binacional y con su devenir cotidiano y menor donde el habitar fronterizo está presente en su devenir diario pero que muchas veces también parece ser imperceptible.

\section{Nación, nacionalismo y nacionalismo banal}

Es reconocible que la nación y los productores del nacionalismo buscan una totalidad expresiva. Sin embargo, aún siendo esa su intención, la evidente finitud de la nación, en tanto sistema de significación cultural, le impide alcanzar tal homogenidad, por lo que su tarea es siempre mantener y actualizar las vías y las estrategias a través de las cuales pueda sostener la mirada en ese horizonte común, buscando dar fuerza a la ilusión de la identidad, como una suerte de "sueño homogéneo" (Bhabha 2010). En ello, la cultura nacional está en permanente contradicción y ambivalencia y afloran de manera constante sus porosidades, sus fugas y sus miedos. Así, y esto es clave para comprender la narración de (y desde) la nación, aquella cultura nacional "crea" y "define" espacios marginales, aislados o no incorporados aún al proyecto como necesidad ineludible para sostener el discurso de la Modernidad o del Desarrollo y, por ende, la vigencia o la solidez del proyecto Nación. Es decir, existe, es o adquiere sentido en la medida que hayan "pendientes": "explorar" para el progreso, "incorporar" para la modernidad o "producir" mayor riqueza para volverse desarrollados, todos engranajes de un mismo panorama discursivo'.

Tal hilo teleológico es, en el fondo, su excusa. Bajo la misma lógica, la nación (re) constituye en forma permanente un ámbito internacional, un "otro" externo, para otorgar racionalidad a "lo nacional". En un caso, expresará que lo "externo" o "internacional" es una imagen con la que la nación debe dialogar e incluso imitar, como sucede por ejemplo, con los países europeos o Estados Unidos. En otro caso, será una amenaza con la que no hay que "transar", como se observa por ejemplo, con Bolivia (Núñez et al. 2014).

Benwell $(2014,2017)$ explora cómo se manifiesta estos discursos en las regiones polares como en el caso de la Antártica. Margarita Serje (2005) ha desarrollado un gran trabajo en torno a este eje de análisis. 
De este modo, la configuración estratégica del territorio común nacional busca dibujar el sentido hogareño que permite alimentar el sueño, el "mito" nacional en ese espacio homogéneo que interpela a sus habitantes. En otras palabras, es precisamente la representación de la vida social bajo los parámetros de lo nacional lo que termina narrando la pertenencia y adhesión, fidelidad que es, al final, más cultural que política:

\begin{abstract}
La nación llena el vacío dejado por el desarraigo de las comunidades y las familias y transforma esa pérdida en el lenguaje de la metáfora. La metáfora transfiere el sentido del hogar y la pertenencia a través de distancias y diferencias culturales que separan la comunidad imaginada del pueblo-nación (Bhabha 2011: 176).
\end{abstract}

Desde este punto de vista, la fabricación de las identidades nacionales desde estructuras simbólicas es lo que le da fundamento al proyecto nacional:

\begin{abstract}
No puede haber nada que sea para la sociedad si no se refiere al mundo de las significaciones, pues todo lo que aparece es aprehendido de inmediato en ese mundo, y ya no puede aparecer sino se le considera en ese mundo. La sociedad es en tanto plantea la exigencia de la significación como universal y total, y en tanto postula su mundo de significaciones como aquello que permite satisfacer esta exigencia (Castoriadis 2010: 557).
\end{abstract}

Así, la producción de imaginarios geográficos es, a diferencia de lo que comunmente se cree, una tarea "sólida" y robusta (Castoriadis 2010; Said 2010; Zusman 2013).

Aquella pretensión de universalidad y totalidad es uno de los pilares centrales de la nación y el nacionalismo en tanto va produciendo sentidos o procesos identitarios de manera constante, buscando evitar la conciencia de su transitoriedad (histórica) o, como dijimos, finitud. Así, una Historia y una Geografía Nacional, la Patria, el Pueblo, la Tradición, entre otras, constituyen mecanismos y horizontes a partir de los cuales se homogeniza la comunidad nacional. Muy relevante es constatar que tales elementos simbólicos deben conjugarse en un marco evolucionista, lineal y teleológico, de modo que la nación vaya narrando, fabricando y proyectando la necesidad comunitaria de sus miembros (Bhabha 2010: 13). De allí que lo simbólico y cultural -lo imaginario, en definitiva- sea más sólido que lo político y es allí donde debe buscarse, desde nuestro punto de vista, los cimientos de lo nacional y los nacionalismos.

\section{Este sistema de significación cultural} llamado nación instala, por tanto, modos de disciplinamiento social que parecen desenvolverse con omnipresencia. Sin embargo, más allá de los productores que podríamos llamar "oficiales", proliferan prácticas y modos de vida que intervienen la noción de nación y nacionalismo "desde abajo". Esta relación -y este concepto es vital para comprender el asunto- supone una re-apropiación de los artefactos simbólicos que la nación busca proyectar en ellos en tanto ciudadanos de la nación comunitaria. Al respecto, el concepto de nacionalismo banal resulta útil para buscar comprender la dimensión nacionalista de escala cotidiana. El nacionalismo banal, término acuñado por $M$. Billig, se refiere a las prácticas donde se expresa la nacionalidad en lo mundano, actitudes y acciones que se dan por sentado y pasan inadvertidas debido a su omnipresencia en lo cotidiano (Billig 1995). El concepto se centra en las formas en la que la nacionalidad se reproduce a través de actos y representaciones comunes que, sin llamar la atención, "marca" el sentido de lo nacional en esa escala menor o mínima (Benwell 2016, Benwell et al. 2018) (Imagen 1). 
Imagen 1. Conmemoración 21 de mayo 2018

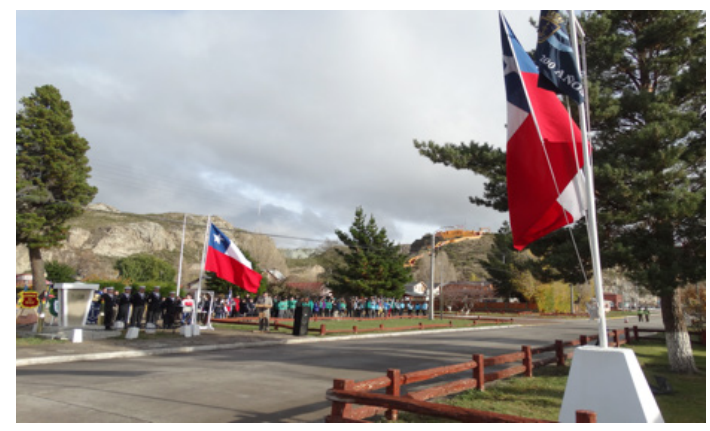

Fuente: Los autores

Así, las distintas imágenes producidas desde la escala nacional son experimentadas desde lo cotidiano para ser re-confeccionadas desde su convivencia cotidiana. En este contexto, lo nacional sale de la escala de poder en la que normalmente se la instala así como también de la posición geopolítica o ideológica que supone la producción de la nación. Por el contrario, lo nacional o el nacionalismo se refuerza y se autoproyecta especialmente desde lo cotidiano, produciéndosey reproduciéndose día a día(Jones y Merriman 2009). Surge así una geopolítica de lo cotidiano. La geopolítica, tradicionalmente instalada en la escala nacional y global, pensada desde los Estados y relaciones de conflicto y tensión, es repensada desde la geografía crítica a partir precisamente de la escala de las prácticas y lo cotidiano (Benwell 2014). Particularmente útil e interesante, el concepto de geopolítica de lo cotidiano interpela a preguntarnos por dónde y cómo se traduce la nación y lo nacional en escalas menores y en zonas, como el caso de Chile Chico, donde la frontera es parte de su campo de significado cotidiano.

\section{La representación de lo nacional en un lugar de frontera: Chile Chico / Los Antiguos}

En la actualidad, donde la movilidad de capitales a nivel mundial ha proyectado una imagen que supone una aceleración del ritmo de la vida, donde todo pareciera reducirse, donde las fronteras se reinterpretan como poco sólidas y donde la compresión espacio-temporal se torna bastante exacerbada, el sentido del lugar, la localidad o la escala menor pareciera perder su importancia. Para la geógrafa Doreen Massey, el resultado se traduce en una creciente incertidumbre del significado de lugar, siendo "visto para algunos como algo necesariamente reaccionario" (Massey 2012: 113), debido a que esta búsqueda de sentido de lugar puede conllevar ciertos efectos como nacionalismos extremos, la recuperación de patrimonios desde una visión sentimental, y un rechazo a los recién llegados o afuerinos. Lo cierto es que a medida que la visión se ajusta a una escala más detallada, es posible reconocer la sobrevivencia de estos lugares, no como un ente inerte y estático, sino más bien, como procesos dinámicos en los cuales se contraponen y superponen distintas relaciones y flujos, a distintas escalas y en constante cambio, que van dando forma a dichas localidades.

Tal dinamismo y movilidad es posible observar en las localidades de Chile Chico (Chile) y Los Antiguos (Argentina) (Ver figura 1). Este lugar ha ido mutando con el tiempo, aunque el tema de la nación y el nacionalismo ha sido una constante, entre otros aspectos, porque allí se conjuga tal vez de modo muy particular la trayectoria de la narración nacional a la cual pertenece, es decir, Chile, mas por otra parte, sus prácticas y su mundo cotidiano dialogan con una memoria que los arraiga a la nación vecina, es decir, Argentina. 
Es importante resaltar que lo que separa a ambos poblados es uno de los íconos clave de todo nacionalismo: el Paso Fronterizo Río Jeinimini- Chile Chico (Imagen 3), que refleja la presencia sólida de la nación en el área. En efecto, la materialidad del paso fronterizo actúa como agencia que viene a reensamblar la acción social conjugándola con la memoria nacional, reforzando en cierto modo, el límite de sus posibilidades (Latour 2008; Lussault 2015). En paralelo, el paso fronterizo muestra una interesante movilidad principalmente por la integración vecinal entre localidades de ambos países, sirviendo al intercambio de las dos poblaciones y al tránsito turístico, habiendo poco transporte de carga, pero un tránsito significativo de pasajeros (Ministerio de Obras Públicas de Chile y Ministerio de Planificación Federal, Inversión Pública y Servicios de Argentina 2012). Los escasos kilómetros que separan una ciudad de otra y el clúster de cerezas que comparten potencia el flujo de residentes, donde además no existen barreras físicas significativas (Manterola 2009).

La presencia de los estados chileno y argentino se materializa a través de las aduanas que se encuentran en este paso fronterizo. La rigidez de la vigilancia permanente resulta algo reciente, siendo ahora más complejo el paso que hace décadas. A pesar de aquella rigidez, desde el Liceo "Luisa Rabanal Palma" de Chile Chico nos comentan:

\footnotetext{
"Este concepto de frontera se diluye también en algunos aspectos porque uno comparte mucho, familia, lazos, amistades, negocios, economía, una serie de cuestiones, compartimos casi el mismo camino, si esta calle principal que cruza es una calle principal de allá (...) y termina, en la ruta 40 , en el norte de Argentina, siguiendo por esta misma calle. Es bien especial esta relación" (V.D., 2018).
}

Imagen 3. Paso Fronterizo Río Jeinimeni

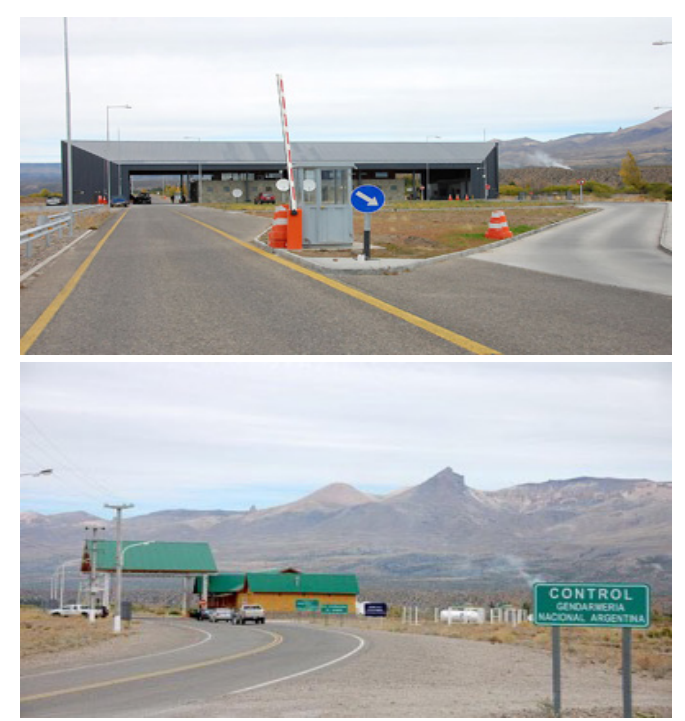

Fuente: InterPatagonia.com, s.f.; PatagoniaAzul.com.ar, 2018.

Los inicios de Chile Chico como pueblo remiten a comienzos del siglo $\mathrm{XX}$, teniendo una historia de poblamiento en común con Los Antiguos, es decir, poseen una raíz similar. Sostienen desde ese entonces una relación de intercambio de especies comerciales y de lazos sanguíneos, además de la cercanía física ya mencionada. Esta relación de inmediatez y familiaridad se mantiene hasta el día de hoy a pesar de una mayor solidez administrativa de la frontera, comprensible desde los contextos geopolíticos estatales de la dictadura militar chilena. En los últimos años, la presencia de fiebre aftosa en Argentina (2001), la implementación de un nuevo seguro obligatorio para pasar en vehículo a Argentina y la movilidad en materia de tipo cambiario en el país vecino, han llevado a que el rol de la aduana se torne cada vez más protagónico. 
Lo nacional, representado en Chile Chico por los servicios públicos y por los discursos que vienen del centro, principalmente de Santiago, es identificado por los jóvenes chilechiquenses como una imagen a la que aspira seguir Chile Chico, donde se se buscaría "ser como el resto de Chile, mientras que allá (en Santiago) se está tratando de imitar al resto de los países del mundo" (C.C., 17 años). Esto nos parece muy elocuente, porque, en definitiva, los propios jóvenes entrevistados (Focus Group, mayo 2018. Imagen 4) constatan que existe una matriz interpretativa que, surgida desde el centro político, los define y los va narrando desde esa escala. Aunque, como ha sido expresado, tal proyección nacionalista es intervenida a partir de su propio espacio existencial. Desde este contexto, surgen 2 grandes factores que marcan y median la relación de esos jóvenes chilechiquenses con el sentimiento nacional: (a) la experiencia de (auto) representar a Chile Chico como un lugar "aislado" y (b) la imagen que se tiene del "norte".

\subsection{La experiencia del aislamiento}

El concepto de aislamiento solo en los últimos años ha venido siendo desmontado de una interpretación monopólica que establecía que todo lo que estuviese alejado del centro político, remitía a marginalidad o a un estado de aislamiento complejo. Desde este punto de vista, ha sido posible constatar que el aislamiento en general responde a un proceso institucionalizado, generando en la práctica un imaginario geográfico donde el aislamiento se presenta como el antónimo de integración y sociabilidad, donde ambos conceptos presentan una carga positiva en el marco de la constante configuración de los estadonación a que hicimos mención con antelación
(Núñez 2011, Núñez et al. 2014; Amigo 2017). Como también expusimos, esos imaginarios actúan de modo sólido y, por lo mismo, quedan impregnados en el horizonte de la comprensión de sus habitantes. En Aysén, ya desde los exploradores de inicios del siglo XX, pero especialmente bajo la declaración de la zona como Frontera Interior durante la dictadura militar, la idea de un territorio aislado y poco integrado marcó su devenir, llegandose a asociar al concepto de aislado con el de soledad, esfuerzo y, sin duda, el de colonización, con todo lo que ello implica².

La conexión de Chile Chico con la capital regional Coyhaique (donde se encuentra la mayoría de los servicios públicos y privados con los que cuenta la región) implica, a pesar de su relativa cercanía, varias horas de viaje, sobre todo si el transporte es por tierra. Por una parte, se puede rodear el Lago General Carrera por territorio chileno en una ruta que se hace larga y peligrosa durante el invierno. Por otro lado, se puede llegar por territorio argentino, donde el camino presenta una relativamente mejor calidad, lo que permite acortar las horas de viaje. Sin embargo, la opción más recurrente de los pobladores chilechiquenses para llegar a Coyhaique es cruzar a través de la barcaza La Tehuelche, que permite atravesar el Lago General Carrera y de la que muchas veces se depende para atender urgencias médicas o realizar trámites.

\footnotetext{
El trabajo de Catalina Amigo (2017) es muy elocuente en demostrar que si el aislamiento se des-institucionaliza y se trabaja desde el habitar el panorama cambia completamente. De hecho, la frase "¿Lejos? ¿Lejos de qué? Ellos están lejos" expresada a Amigo en su trabajo de campo, resume las "otras" posibilidades de sentido y comprensión del aislamiento.
} 
Imagen 4. Focus group en Liceo Luisa Rabanal Palma

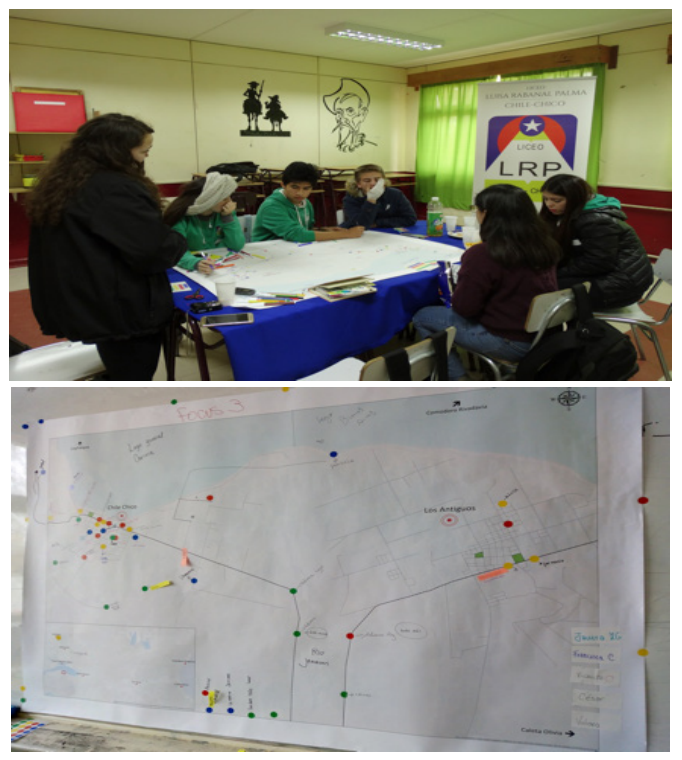

Fuente: Los Autores

De este modo, los y las jóvenes de Chile Chico reflejan esa lejanía de lo que implican los centros de poder, tanto Santiago como Coyhaique:

Yo creo que de aquí hacia abajo se vive realmente lo que es el aislamiento, porque hasta Coyhaique como que llegan cosas. Quizás, en lbáñez igual están como aislados, pero yo siento que acá se vive más el aislamiento" (F.C., 16 años).

\section{Complementando esto, otro estudiante expresa:}

Estamos en un país aislado, en la región más aislada y en uno de los pueblos más aislados de la región. Estamos tan aislados... porque el pueblo más cerca es Guadal, para ir es como mínimo 1 hora, porque el camino nunca está bueno y es peligroso (...) La barcaza es la principal conexión entre nosotros y lo que sería "Chile" (V.O., 16 años).

De esta forma, la barcaza es la principal conexión que se tiene con el resto de Chile, que como veremos denominan "el Norte". La Tehuelche no sólo se presenta como un medio de transporte necesario para llegar al principal centro regional, también se identifica como un elemento explícito de la presencia del Estado chileno en esta zona, que llega a dar respuesta al sentimiento de abandono que se ha tenido en relación a la nación:

\begin{abstract}
Y hasta el día no hay una conexión con el resto de Chile para arriba y ni siquiera a nivel medio. Es decir, tengo que ver Televisión Nacional para que salga Chile Chico porque en otro canal no sale, porque no existe (...) uno empieza a compararlo con Chiloé, y no sé, dicen: "queremos un puente que nos conecte porque estamos aislados" y uno dice "no conocen el aislamiento"... Tienen una barcaza que pasa cada media hora allá y acá una vez al día y depende de condiciones climáticas (C. A., 2018)
\end{abstract}

Otra de las formas en las que se traduce y expresa la experiencia y tradición de aislamiento se asocia a los altos costos de vida que deben asumir los habitantes, tanto para conseguir una hora para atención médica con especialistas en Coyhaique y el elevado valor de los productos de consumo diario:

La leña el metro treinta mil pesos (...) Lo que significa además comprar todo lo que es comida en el supermercado y sale alrededor de ciento veinte mil pesos para dos personas (...) Para todo, nosotros, todo llega como tarde. La moda de ropa es tarde. O sea, bacán verlo en la tele... pero que llegue acá... Entonces es súper complejo, es ingrato. Lo comparo mucho con cómo se sienten las comunidades indígenas. Totalmente desplazados y totalmente aparte de este Chile que solamente, y eso yo creo que es un sentimiento generalizado, que solamente existe en Santiago y en las capitales regionales (P. H., 2018)

Uno de los impactos de esta experiencia del asilamiento en Chile Chico es que en muchos casos cruzan a Argentina para comprar mercadería o asistir a los servicios de salud que además son gratis. De este modo, la noción de 
aislamiento se encuentra institucionalizada en la medida que la nación se asocia a Santiago y a los centros regionales que son representativos del poder central. Este modo de comprensión, impactado por su dimensión material, genera una doble proyección social. Por una parte, la nación se instala como un asunto de conflictividad en tanto "lejanía" y, por otra, se ve resaltada la sensación de pertenencia a la comunidad nacional en tanto zona excepcional, lo que en la práctica es traducido como "haciendo Patria".

\subsection{El Norte y el límite de la Patagonia}

En paralelo a la frontera material del Paso Río Jeinimeni- Chile Chico que separa Chile de Argentina, es posible observar una frontera simbólica que acompaña la proyección de aislamiento a la que ya hemos hecho alusión. En efecto, se identifica a Chile Chico, y a la región de Aysén en general, como el 'resto' del país que difiere con el Norte. Así, para las personas de Chile Chico el límite norte se encuentra en Puerto Montt, produciéndose una diferenciación desde un sentido cultural que los identifica hacia el sur de este límite, el que también coincide con la delimitación que muchas veces hacen de "la" Patagonia (Figura 1).

De este modo, todo lo que se ubique más allá de Puerto Montt no formaría parte de su zona de pertenencia, al menos más inmediata:

\footnotetext{
El truco es un juego que se juega en la Patagonia, no se juega en Santiago ni en lquique, claro, lo juegan en Valdivia o en Temuco, pero ¿quiénes lo juegan? Los chicos de la Patagonia, de Chile Chico. Acá casi todos los niños juegan truco (V.D., 2018).
}

Ello concuerda con lo expresado por una profesional local que indica que:
Los jóvenes se sienten identificados con cosmovisiones muy marcadas, muchos no soportan el cambio de hábito y de costumbre 'fuera de casa', por lo que si salen a estudiar, es a las ciudades más cercanas, donde se sienten aún cerca de la Patagonia (N. O., 2018).

Por otra parte, en los y las jóvenes consultados dentro de los focus group, se evidencia un arraigo importante con su tierra incluso si "se va a estudiar al norte para luego volver al lugar de crianza, de origen". La identificación con la Patagonia surgiría también como una necesidad de diferenciarse:

\footnotetext{
De pronto hay una moda de ser patagón, de andar emboinado y decir "soy de la Patagonia". Y es como el plus, casi una marca, tal cual como que vende bien eso de ser de Patagonia y con esto que es la gente de acá la que escribe, que lo de afuera está mal (...) como de este chovinismo exacerbado... sí, porque son nacidos ahí (C. A., 2018)
}

En lado argentino también se daría esta dinámica, una identificación con la zona, lo que se traduce también en las relaciones sociales:

\begin{abstract}
El argentino quiere mucho al sureño, de Puerto Montt para acá, pero al nortino no lo quiere mucho. Lo mismo pasa, que el argentino sureño no quiere al argentino norteño (...) hay un tema con la cercanía (...) Son similares, lo que pasa es que el lugar de fronteras muy cercanas desde Punta Arenas, Valdivia, todo ese lado para acá... Entonces la gente chilena va a trabajar al otro lado (F. A., argentino, 2018)
\end{abstract}

Tal frontera simbólica, en definitiva, colabora a producir topofilia, es decir, una arriago y reconocimiento con el lugar (Fu Tuan 1974), lo que, del mismo modo que el aislamiento, los lleva a reafirmar que a veces la nación está lejos aunque por otra parte esa es su comunidad imaginada proyectada desde su propia particularidad. Como nos fue expresado: "La región tiene un ritmo distinto al del país (...) lo cotidiano y las distancias son totalmente distintas" (C. A., 2018). 


\section{Nacionalismos cotidianos: una expresión de la memoria}

La memoria es un factor relevante para los propósitos de esta investigación en tanto actúa como propulsora del arraigo, de la pertenencia al lugary también como elemento clave al momento de la construcción del ciudadano nacional. En este caso, la relación de la comunidad de Chile Chico con la imagen de lo nacional ha variado a través del desenlace de distintos procesos históricos, como también han intervenido los constantes eventos de tensión-armonía con el otro lado del borde nacional.

En esta zona, la relación entre ambos países tiene su origen a inicios del siglo $X X$ con la llegada de colonos a Chile Chico, provenientes de una larga travesía desde Antuco, zona del Bío Bío (Chile), con destino a Argentina en búsqueda de una mejor calidad de vida. Por presiones del gobierno argentino, la mayoría de esos migrantes avanza insistentemente hacia el sur en busca de territorios chilenos desocupados. Ya instalados en lo que denominaron "Chile Chico", nombrado así por la nostalgia de reencontrarse con la patria, mantienen el contacto constante con el territorio argentino, no sólo por tener aún familiares en dicho país, sino también por los largos viajes que realizaban para poder abastecerse de insumos básicos (Ivanoff, 2013). En la práctica, si bien había un Chile al norte y su mirada era norte-sur (vertical) su mundo y sus movilidades ordinarias era con el territorio argentino, es decir en un sentido oeste-este (horizontal) ${ }^{3}$.

Para el año 1918 se produce el primer suceso de tensión entre los pobladores de Chile Chico

Respecto de estas prácticas horizontales ver Núñez 2013 y Núñez et al 2017; Carrasco 2017). con el "norte" (Chile), quienes vivían hace algunos años ya en ese lugar. La llamada Guerra de Chile Chico (o los Sucesos del Lago Buenos Aires) se produce al momento de intervenir el interés de inversionistas magallánicos (capital inglés) que respaldados por el Estado chileno buscan instalarse en las tierras ya ocupadas por lo pobladores: "Comienza así a gestarse una historia de enfrentamientos, de dolor y de abuso de autoridad, en la que interviene la fuerza militar de Chile en contra de los pobladores" (Ivanoff, 1996:5). El suceso, marcado por la acción de un grupo de hombres y mujeres que con el anhelo de volver a vivir en su país se enfrenta a las fuerzas de su propia nación para defender sus tierras de la ocupación empresarial, además de ser un asunto muy actual, se menciona como uno de los principales eventos que describen la relación de presión y distensión entre los pobladores y la presencia de lo nacional ${ }^{4}$.

A su vez, los hechos políticos y socioeconómicos han marcado distintas pulsiones en la relación Chile-Argentina y específicamente Chile Chico-Los Antiguos. Las crisis económicas y situaciones extremas como la erupción del Volcán Hudson (1991) y siniestros como incendios producidos en ambas localidades donde se coopera con ayuda mutua, han mostrado la colaboración-tensión entre ambas naciones. Por una parte, a partir de la inminente guerra entre Chile y Argentina (1978), todavía hacia 1984 se manifestada aquella expresión de un nacionalismo exacerbado: "Había ejercicios militares acá, la población escolar, los docentes,

En la actualidad se está dando un proceso con un fondo similar a partir del arribo de grandes capitales de tipo conservacionista que junto con concentrar la gran propiedad de Aysén han colaborado sustancialmente en cambiar el destino agrícola y ganadero de la zona con todo el impacto que ello implica para los habitantes tradicionales locales (Núñez, 2019) 
nos tomaban el ejército y... ejercicios militares acá arriba del cerro, prácticas de combate y todo eso" (R. M., 2018)

Por otra parte, sin embargo, la memoria de un espacio común y de cooperación recíproca entre Chile Chico y Los Antiguos es por lejos la que más sobresale. Un caso particular fue lo ocurrido durante el año 1991 con una de las más violentas erupciones del Volcán Hudson:

\begin{abstract}
Yo creo que cuando ocurrió el tema del volcán acá en Chile Chico, nosotros resentimos mucho el trato que nos dio el gobierno, porque en Argentina a todas las familias se les dio una canasta familiar, se les dio leche, acá, nada (...) Muchos, cuando estuvimos en el gimnasio, levantaron la bandera de Argentina y pensaban cambiarse de nacionalidad porque veían que al otro lado los estaban atendiendo, me parece que vino un destacamento del otro lado y ofreció ayuda para acá y de acá las autoridades dijeron que no... pero es cierto, aquí se sacó arena desde los techos de las casas... pero como familia, nada (R. M., 2018. El subrayado es nuestro).
\end{abstract}

En la memoria del habitante chilechiquense se encuentra este recuerdo y es latente, tanto en los jóvenes como en los más adultos. Para los jóvenes, la memoria se representa muchas veces a través de los abuelos, en tanto son ellos los que cuentan anécdotas entre Chile y Argentina. A una de las entrevistadas, su abuelo le cuenta que luego de la erupción del volcán las primeras máquinas que llegaron a despejar calles fueron argentinas, seis meses después llegó la ayuda chilena, lo que explica una especie de resentimiento entre las personas mayores con Chile:

Las personas del norte creen que porque vivimos al lado de Argentina es como traición, pero si uno ve a las personas de Chile Chico, son bien nacionalistas, defienden su patria y todo. En parte yo creo que si uno hace una encuesta a los más viejitos, tienen un resentimiento a su propio país, como que siempre Chile los deja solos al final, es como un lugar muy apartado (M.S., 16 años).

\section{La escuela, lo doméstico y el hacer en la producción del ciudadano nacional en Chile Chico}

El ciudadano nacional es entendido como el individuo perteneciente a una nación con la cual se identifica y es a la vez una producción de los discursos dirigidos por el Estado y las clases gobernantes, donde diariamente en pequeñas cosas que son desapercibidas se les recuerda aquella pertenencia a una nación (Billig 1995). Si bien, la decisión de ser ciudadanos nacionales no es consciente, con el tiempo el individuo se encuentra, como hemos visto, reclamando activamente una identidad nacional, la que es proyectada de forma consciente (Dittmer y Doods 2008; Antonsich y Skey 2017).

Su formación como ciudadano nacional se lleva a cabo mediante diferentes mecanismos y experiencias, donde la memoria y la proyección imaginaria influye en el sentido de pertenencia o no con una nación, pero también estos mecanismos están representados en las instituciones públicas, las escuelas (educación formal) y la familia (lo doméstico), el hacer cotidiano, las ceremonías conmemorativas, entre otras. De esta manera, se entiende que la identidad nacional, el ciudadano nacional, está en constante construcción y a pesar de ser menos perceptible, el proceso se desarrolla en y desde lo cotidiano.

\subsection{La educación como mecanismo de producción de identidad nacional}

El rol de los establecimientos educacionales en la transmisión de la identidad nacional es esencial en la articulación social de la proyección del ciudadano nacional. El papel de la educación está en buena parte dirigido por el 
Estado a través del mantenimiento y supervisión de la infraestructura social, que tiene por objetivo "producir a miembros de la sociedad responsables, leales y competentes" (Gellner 1983: 90). Sin embargo, y esto es clave para entender la producción del ciudadano nacional, no solo el disciplinamiento es indispensable sino la necesidad que el individuo avance en el autogobierno de modo que la pedagogía nacionalista sea una formación identitaria permanente (Deleuze, 2006. El subrayado es nuestro). Desde este punto de vista, siguiendo a Foucault (2012) y Deleuze (2006), el disciplinamiento que construye la escuela no solo es forzado desde regulaciones y reglas, de hecho, este aspecto es el menos robusto, sino que se transforma en un proceso virtuoso de (auto)producción de realidad donde la conducta es encauzada a nivel microscópico para reconocer (se) en los símbolos que la nación transmite en la escala menor o cotidiana:

\footnotetext{
La escuela, crea un verdadero y constante intercambio de saberes: garantiza el paso de los conocimientos del maestro al discípulo, pero toma del discípulo un saber reservado y destinado al maestro. La escuela pasa a ser el lugar de elaboración de la pedagogía (Foucault, 2012, 217)
}

En Chile Chico, históricamente el Liceo Luisa Rabanal Palma y la Escuela Básica de Chile Chico han sido los encargados de proveer y cubrir la necesidad de una identidad nacional, no sólo por lo que se enseña en sus aulas, también por instancias como la Banda Chile Chico (años 70, banda instrumental), Celebración de Fiestas Patrias, Conmemoración de las Glorias Navales (actividades en liceo y desfile comunal), entre otras actividades concretas. Dentro del currículum académico centralizado entregado por el Ministerio de Educación la Historia de Chile (con mayúscula) y desde allí la enseñanza de una memoria y símbolos patrios se encuentran en la mayoría de los niveles, siendo fuerte durante los primeros años de formación. Por el contrario, los contenidos sobre una historia local no son incorporados en el currículum. De acuerdo a lo expresado desde el trabajo en el Liceo, la historia local, por ejemplo, todo lo referido a la ya comentada Guerra de Chile Chico, no tiene cabida curricular en tanto sus directrices son centralizadas predominando el paisaje de una memoria común a la nación:

\begin{abstract}
Hace muchos años atrás el currículum sí tuvo un momento en que la asignatura de historia comenzaba con la historia local (...) Era un programa bastante bueno, partía de lo micro a lo macro, pero desaparece... Lo cambiaron. Mientras duró, poco, fue bien bueno porque los chicos hacían exposiciones de su realidad, su cotidianidad, de sus paisajes... Pero después ya no, el ministerio aprobó otro programa y empezó a aplicar ese de escala nacional (V. D., 2018)
\end{abstract}

En el trabajo de campo fue posible observar que el conocimiento que los jóvenes manejan sobre historia nacional e historia local, difiere mucho entre generaciones. Algo en apariencia tan natural como el reconocimiento del entorno en el que se vive puede aparecer o desaparecer de acuerdo a las decisiones tomadas desde un poder político central, ligado también a un periodo político. A su vez, el ciudadano nacional de hoy se ve marcado directamente por la educación que recibieron sus padres $y$ el contexto en el que ella se dio, ya que es a través de la institución de la familia, como veremos, donde también se despliegan los símbolos identitarios nacionales. F.A., por ejemplo, de 15 años, cuenta que en su caso sus padres no tuvieron la oportunidad de completar sus estudios, por lo que cree que por eso las enseñanzas sobre lo nacional no las recibe de parte de ellos, sino de la escuela y del liceo. 
Por otra parte, la historia local y la divulgación de ésta va a depender exclusivamente de la intencionalidad de la administración del establecimiento y de la voluntad de los profesores. En el caso del Liceo nos comentaron que existen instancias, como el Proyecto Institucional (PI):

Donde pueden desplegar contenidos de una memoria local o también durante las asignaturas de orientación, consejo de curso o integrándose dentro de la programación de algún otro ramo, dependiendo de la voluntad de cada profesor" (V.D., 2018)

Sin embargo, estos espacios aparecen solo de modo excepcional porque la estructura del saber educacional presenta un predominio de la escala nacional.

En el devenir de las prácticas locales se conjugan y fusionan, por tanto, horizontes de comprensión de diversas escalas. Lo relevante, desde el plateamiento de este trabajo, es que aquella fusión, si bien híbrida, también manifiesta jerarquía de valores. Por un lado, se reconoce el arraigo a la memoria nacional, deseándola, necesitándola (el paso del inconsciente al consciente), mas por otro, se despliegan y deslizan trayectorias que componen una grafía de una tierra (geo) que arranca desde lo cotidiano, desde las prácticas, y donde menos tiene que ver la escala universal u homogénea. Como ha planteado De Certeau, una sociedad se compone de prácticas que organizan un discurso, que normalizan o disciplinan pero también "de otras prácticas, innumerables, que siguen siendo menores, siempre presentes ahí..." (De Certeau 2010: 56).

Lo expuesto con antelación se observa con radical claridad desde nuestro punto de vista, al constatar la existencia de un carácter "binacional" en la composición de cada grupo escolar sea éste de Chile Chico o Los Antiguos.
Así, "es normal ver en la sala de clases una identidad 'binacional'....está normalizado" (J.D., 2018). Incluso, de acuerdo a la misma fuente, hace algunos años atrás la baja cantidad de alumnos argentinos matriculados en Chile Chico fue algo que llamó la atención, por lo irregular del asunto. Sin embargo, "para el año 2018 se registró un aumento en las matrículas de estudiantes trasandinos" (P.H., 2018), por lo que "hoy es normal que en la sala de clases surjan temáticas de Argentina como lo sucedido en Malvinas, ya que viene con la identidad de los jóvenes" (J.D., 2018).

En la práctica, en la cotidianidad, Chile Chico es uno de los pocos lugares donde se enseña historia chilena e historia argentina, y es así porque el aula lo pide. Cuando se habla de la dictadura en Chile también se habla de Videla; cuando pasan geografía del territorio, también se habla de la geografía de Argentina. Para el profesor esto es algo inevitable porque es lo que "los jóvenes conocen y lo que está en su experiencia" (J. D., 2018. El subrayado es nuestro). Es decir, la comunidad imaginada de Benedict Anderson es exportada en forma independiente de la nación de destino.

\subsection{El papel de la familia: el traspaso de la identidad en (y desde) lo doméstico}

Bachelard desarrolla una hermosa idea que, en cierto modo, desnuda los pretendidos cimientos científicos y universalizantes de los estudios geográficos. Expone que es posible considerar una casa como un "objeto", como algo formal que se puede describir y exponer desde su positividad. Más, se pregunta, ¿dónde queda el "espacio interior", el habitar del hogar, "el noyo que protege al yo"? (Bachelard 2010: 35). En otras palabras, ¿dónde queda la experiencia 
de la casa? ${ }^{5}$ En este contexto, considerar lo doméstico, el plano familiar, como unidad de traspaso de prácticas, memoria e identidad, es fundamental para el proceso de formación del individuo nacional y para comprender la geograficidad de los habitantes de Chile Chico (Dardel 2013). En efecto, el rol de la familia en la divulgación de una memoria local/nacional es clave.

Según el testimonio recopilado del trabajo con los y las jóvenes, los abuelos, como principales actores de la tradición oral, son los encargados socialmente de divulgar las historias que dicen relación con la identidad chilechiquense, siendo muy importante dentro de la consolidación de una identidad local. A pesar de su importancia, esa configuración de memoria se va movilizando con el tiempo y las nuevas generaciones ya no transmiten los mismos horizontes que sus ancestros. La imagen de sus padres, ya no de sus abuelos, en cuanto a la entrega de información a través de relatos orales no es en la actualidad un valor en sí mismo. En cierto modo, "esas historias" representan un pasado menos presente, menos protagónico que en décadas previas (Imagen 5). Recordemos que ellos fueron formados bajo la impronta de la dictadura militar y por ende de un disciplinamiemto muy fuerte en torno a lo nacional.

En efecto, un testimonio nos permite asociar temporalidades donde la producción del

\footnotetext{
Esta idea es replicada por el geógrafo francés Eric Dardel del siguiente modo: "La geografía no es, en principio, un conocimiento; la realidad geográfica no es, ante todo un objeto; el espacio geográfico no es un espacio en blanco que se rellene coloreándolo (...) es decir, la Tierra no es un dato bruto a considerar tal como ella se presenta, sino que entre el hombre y la tierra siempre se desliza una interpretación, una estructura y un horizonte del mundo..." (Dardel 2013: 94).
}

Imagen 5. Mural en honor a los pioneros

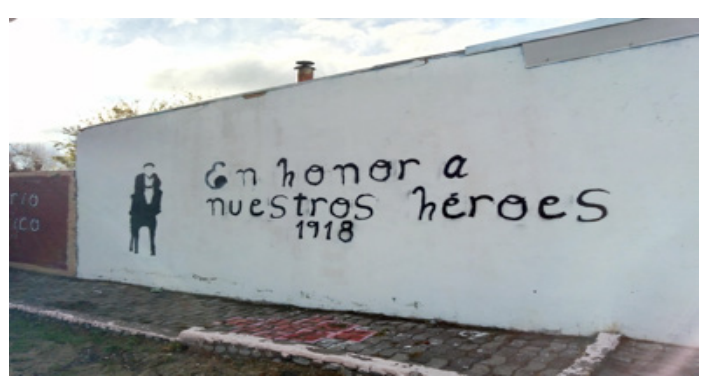

Fuente: Los autores

ciudadano nacional se torna más sólida que en otros momentos:

\begin{abstract}
Es como una generación entre los 40 y los 55 años, es una generación que no engancha, hay como una brecha de edad que no aparece, quizás tiene que ver con los procesos políticos de un país, como modo de justificar ese vacío (C. A.,2018).
\end{abstract}

Esa generación a la que hace referencia la entrevistada es la crecida y formada en el período de la dictadura militar (1973-1989). Cabe mencionar que en ese período, tal vez como en ningún otro, las lógicas geopolíticas del Estado se instalaron con fuerza en la zona llegando incluso a ser declarada como una Frontera Interior, es decir, un espacio dónde con especial atención debía actuar el orden nacional, dada su aún precaria incoporación a la nación. Es en esa época donde el despliegue de los discursos sobre el aislamiento regional se tornaron más sólidos, traspasando ese (re)conocimiento a los ciudadanos nacionales. En 1982 era posible leer:

\footnotetext{
El vasto sector que constituye la Región de Aysén ha estado históricamente aislado del resto del país y, aún, carente de comunicaciones en su propio territorio a causa de su difícil morfología geográfica, geológica y climática (Dirección de Vialidad, 1982).
} 
Desde esta perspectiva, es posible afirmar que determinados regímenes de enunciación social como lo de escala nacional son capaces de diluir la historia local y el traspaso de la memoria, lo que finalmente impacta en los recuerdos y en las experiencias más íntimas a las generaciones que siguen.

Así, el rol de la familia generará marcas en la memoria de los jóvenes, que los sintetiza con las que son transmitidas por la propia escuela, por los medios de comunicación o por la serie de símbolos nacionales presentes en el quehacer cotidiano, como las réplicas de bailes que reflejan el Chile central como la cueca o las banderas chilenas que flamean constatemente para recordar la pertenencia a la "comunidad nacional" (Imagen 6).

Imagen 6. Conmemoración 21 de Mayo

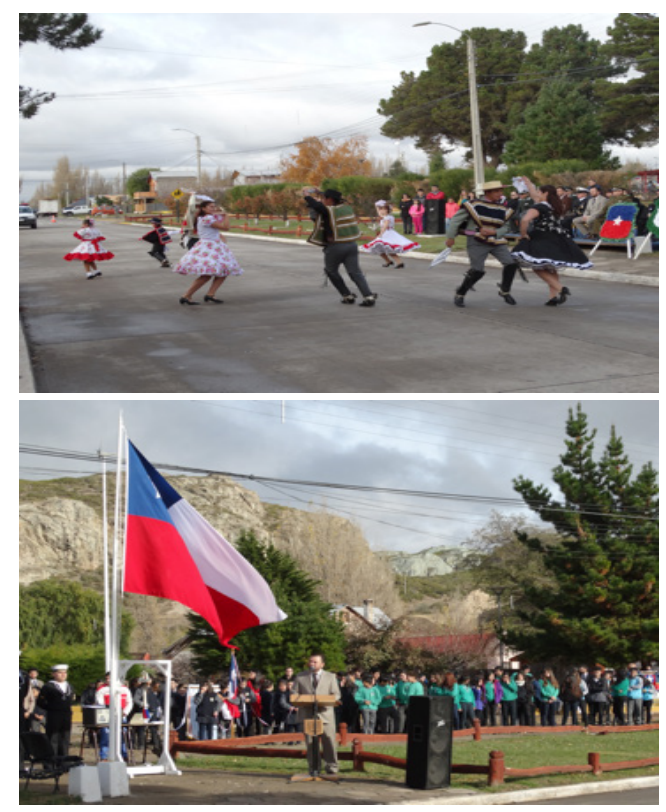

Fuente: Los autores
A su vez, desde este conocimiento adquirido en el ámbito familiar, los jóvenes dialogan con los símbolos que los comprometen con el espacio público como, por ejemplo, monumentos oficiales que conmemoran a los mártires nacionales o murales que representan interpretaciones particulares de la nacionalidad (Colins Collins y Coleman 2008; Benwell 2014).

De este modo, la construcción social de una identidad nacional se ve reflejada principalmente en la celebración de hitos históricos de escala nacional. Las fechas más importantes son el 21 de mayo y el 18 de septiembre, donde, por una parte, se conmemora el triunfo nacional sobre "el enemigo extranjero" $y$, por otra, se celebra lo que podríamos denominar el cumpleaños de la nación, es decir, la fecha de su independencia del imperio español. Antes, sin embargo, en plena dictadura, la relevancia de estas ceremonias era mucho más fuerte:

Antes se veía más... se hacían desfiles, ramadas, se escuchaba cueca, se bailaba. Las familias se vestían con su mejor pinta, nos compraban ropa nueva para la época, había una preparación para ese día (N.O., 2018).

Es interesante constatar a través de este relato como las identidades y los valores asociados a ellas van cambiando y pueden incluso llegar a tener acepciones múltiples y diversas. En cierto modo, en cada identidad, por más sólida que parezca, se instala una pluralidad silenciosa de sentidos que más que evolutivos, actúan y se desenvuelven de manera simultánea (Deleuze 2002). Así, en épocas más recientes, lo patagónico o "La Patagonia" adquiere valor en un contexto global donde la imagen de fin de mundo asociada a la región de Aysén toma fuerza. En cierto modo, la escala nacional dialoga con la global y vacía nuevas representaciones en los jóvenes locales. Por 
ejemplo, se llegan a reconocer en una cultura patagona y gauchesca como valor jerarquizado socialmente:

...hoy en día se le ha dado más valor a la cultura argentina y como "somos los patagones", esa visión se ha hecho más amplia, pero eso es como, yo no sé, diría como hace unos 30 años" (N. O., 2018).

Aquello no suprime, sin embargo, la constante producción y reproducción de lo nacional. En relación a esto es necesario reconocer a estos jóvenes como agentes geopolíticos que colaboran a dar fuerza a la interacción entre fuerzas nacionales, binacionales o globales (Benwell et al. 2018):

Yo como director podría decir: saben, nosotros somos un liceo chileno, aquí no se permite el chamamé, aquí el baile nacional es la cueca y la cueca es lo que vamos a privilegiar, aquí no vamos a desfilar vestidos de gaucho, aquí desfilamos vestidos de huaso. Pero si hiciera eso, yo estaría desconociendo este tipo de pueblo, este tipo de sociedad, que es otra cosa, una mescolanza de cosas. Valoramos lo otro también... tenemos grupo folclórico, hacemos cueca, hacemos danza, pero también valoramos lo que ya está internalizado (V.D. 2018).

Así, la familia campesina o 'campera' es el verdadero nicho de prácticas gauchescas debido a las actividades que realizan para vivir. El clima, el espacio físico, la agricultura y la ganadería, las distancias, la disponibilidad de recursos y el modo de vida en sí, concuerda completamente con dichas prácticas:

"La influencia también la hace la familia, la familia campesina (...) campesinos que tienen metido este tema de lo gaucho, de lo patagón, lo tienen metido en su ADN" (V. D.,2018).

La procedencia de las familias también tiene, por tanto, un alto impacto en la formación de la identidad de los más jóvenes. En algunos casos, esas familias campesinas han intentado preservar la memoria local, ya sea por ser descendientes de antiguos colonos (llamados pioneros) o jugar un rol importante dentro de la comunidad, es decir, se identifica un discurso que se transmite a los más jóvenes y coincide con ello un arraigo muy potente al lugar. Esto se ve reflejado en la forma en la que los jóvenes reconocen su localidad y proyectan su futuro en Chile Chico, a diferencia de los jóvenes de familias que han llegado en las últimas décadas al lugar que presenten una cosmovisión con menos arriago y más anclados en lecturas nacionales y globales.

\subsection{Trayectorias cotidianas: el hacer en un espacio de frontera}

El hacer en un espacio de frontera, es decir, aquellas prácticas ordinarias que se desenvuelven diariamente, co-producen relaciones de sentidos espaciales fronterizos donde conviven las trayectorias de un devenir asociado a la memoria local con uno proyectado desde la comunidad nacional. Estos encadenamientos se manifiestan en operaciones cotidianas, efímeras en muchos casos, desde donde surgen, se olvidan y recomienzan según sea el caso. En efecto:

\footnotetext{
"Mientras que los estados argentinos y chilenos buscan asegurar el territorio nacional a través de mecanismos estrictos de control fronterizo y el establecimiento de "comunidades nacionales", las experiencias y la vida cotidiana de esos ciudadanos que viven en la región fueron y siguen siendo altamente relacionales" (Baeza 2009 en Núñez., Baeza y Benwell, 2017).
}

En el hacer de la frontera en Chile Chico confluyen esos itinerarios temporales del hacer nacional y local, donde lo nacional se organiza y se reconoce y se olvida al mismo tiempo. La movilidad del individuo representada en 
trazados cotidianos y recurrentes crea la imagen de su entorno y de sí mismo. De esta forma, la frontera en Chile Chico es parte de su habitar existencial así como también lo son algunas zonas de la región argentina donde por ejemplo hay fuertes lazos familiares.

Desde el asentamiento de las primeras familias, tanto de lado chileno como argentino, el cruce entre un territorio y otro, atravesando el Río Jeinimeni fue una práctica recurrente. La fluidez de las relaciones en Chile Chico y Los Antiguos lleva a que la barrera se diluya aunque no se desconozca:

"El concepto de frontera es, claro, una frontera política, física también podríamos decirlo porque está en un mapa puesta a través de unas rayas, poligonales, pero se pierden en algún sentido de esta zona especial porque tú tienes amigos, familiares, parientes..." (V.D., 2018).

Con los años y la oficialización de la frontera a través de las aduanas, el tránsito entre un lugar y otro se ha ido tornando más engorroso, más complejo. Si bien sigue siendo constante visitar territorio argentino, últimamente la implementación de aquellos mecanismos de control, según vimos, como por ejemplo, el seguro exigido para los vehículos chilenos que pasan a territorio argentino, ha venido a limitar en parte el flujo: "Apareció un famoso seguro que uno tiene que cancelar, que no es barato y las cosas subieron muchísimo al otro lado, entonces no convenía" (F.C., 16 años). Paradójicamente, la única excepción a los trámites aduaneros corresponde al Cuerpo de Bomberos de Chile Chico y Los Antiguos, facilitando el paso de los carros bomba para acudir en caso de emergencias. Esta excepción nos resulta muy simbólica, porque da cuenta de una espacialidad compartida que interrumpe las formalidades del hacer fronterizo de escala nacional para dar paso a un hacer comunitario de escala local.

Aquella "ruptura" a la formalidad fronteriza por parte del cuerpo de bomberos, de acuerdo a lo recopilado en el trabajo de campo, es el reflejo a su vez de otras prácticas informales como el cruce constante de los límites nacionales por parte de lugareños, argentinos y chilenos, que sin ánimo de desafiar a la autoridad, se desenvuelven en un ir y venir por pasos fronterizos no oficiales donde la experiencia del habitar y las prácticas tradicionales como el pastoreo y las veranadas se conjugan a las imposiciones de las naciones. En cierto, modo, este juego de tácticas silenciosas, a diferencia de lo que podría pensarse, resisten al orden nacional impuesto, pero a la vez, lo avalan y lo registran por omisión en tanto esos mismos lugareños volverán para pasar en otros momentos por los pasos fronterizos oficiales para reconocerse en la comunidad imaginaria de su nación y sentir allí también un lugar de pertenencia, un "hogar".

\section{Conclusiones}

Como ha planteado Massey (2012), no es posible pensar el lugar sin su sentido global. Del mismo modo, estimamos no es posible abordar cuestiones geográficas sin constatar las relaciones de fuerza que hay detrás de cada sentido territorial. No todo estaría disciplinado y controlado, ya que como ha planteado De Certeau (2010), en las trayectorias ordinarias y cotidianas la imagen de los fabricantes de lo nacional es mediada por las prácticas y por geografías menores que se desenvuelven 
forjando horizontes propios y únicos. $\mathrm{Y}$, sin embargo, en coherencia con lo formulado en esta propuesta, esas geografías menores, a su vez, se co-fabrican desde los procesos de control y disciplinamiento de alcance nacional. Desde esta perspectiva, todo estaría relacionado y se desenvolvería de modo múltiple dando paso a una movilidad constante de juego de valores donde lo relevante, al menos en principio, estaría en reconocer la o las acciones que producen el o los elementos diferenciales de ese juego de valores.

Así, el presente trabajo ha buscado indagar, en lo sustancial, en torno a un mundo cotidiano, a una geografía menor en un espacio de frontera que (se) reconoce también desde trayectorias de escala nacional y en forma paralela en formas que en muchos casos poco y nada tienen que ver con lo nacional. En efecto, como vimos, hay prácticas, espacios públicos y memorias que supondrán un reconocimiento estrecho con el orden discursivo emanado desde los centros políticos. Y, por otra parte, los lazos de pertenencia a un territorio construido y proyectado desde inumerables acciones que suponen una superación de los límites fronterizos nacionales fluye diariamente a partir de las diversas relaciones existentes entre las localidades de Chile Chico y Los Antiguos.
De este modo, es posible afirmar que el ciudadano nacional no solo es producido desde el centro político, desde el orden discursivo de índole nacional sino, especialmente, desde su devenir cotidiano, desde su quehacer silencioso y menor que implican sus prácticas, donde ellos auto-proyectan sentidos nacionales desde los dispositivos simbolismos y materialidades presentes en sus respectivos territorios, tales como banderas, murales, ceremonias, la educación, el espacio doméstico o en y desde el hacer de sus propias trayectorias culturales.

En este contexto, para finalizar, es necesario decir que a pesar del relevante grado de abstracción que poseen las estructuras transmitidas por una Historia y una Geografía Nacional, en tanto buscan homogenizar el espacio y la memoria en clave nacional, ellas se impregnan en los ciudadanos en y desde sus prácticas generando un ciudadano nacional que también se refleja en esa comunidad amplia llamada nación. Por tanto, el habitante local se transforma, como hemos expresado, en un agente geopolítico que desde su devenir diario produce y rehace el sentido comunitario y de pertenencia a la nación y a su vez, en agente de una memoria que pone en valor un devenir que les pertenecía incluso antes que llegara la nación y sus límites.

\section{Bibliografía}

Amigo, C. 2017. "No estamos lejos, allá están lejos. Perspectivas locales sobre aislamiento en Aysén: discurso estatal y aislamiento como territorialidad". Imaginarios geográficos, prácticas y discursos de frontera. Aisén-Patagonia desde el texto de la nación. Núñez, A., et al. (Eds.). Santiago: LOM. 167-190.

Anderson, B. 1983. Imagined Communities. London: Verso Books.

Antonsich, M. \& Skey, M. (Eds.). 2017a. Everyday Nationhood.
Theorising Culture, Identity and Belonging after Banal Nationalism. London: Palgrave.

Antonsich, M. \& Skey, M. 2017b. "Introduction: the Persistence of Banal Nationalism". Everyday Nationhood. Theorising Culture, Identity and Belonging after Banal Nationalism, Antonsich, M \& Skey, M (Eds.) London: Palgrave Macmillan. 1-13.

Bachelard, G. 2010. La poética del espacio. México D.F.: Fondo de Cultura Económica. 
Benwell, M. 2014. "From the banal to the blatant: Expressions of nationalism in secondary schools in Argentina and the Falkland Islands". Geoforum. 51-60.

Benwell, M. 2016. "Banal Nationalism". The Wiley-Blackwell Encyclopedia of Race, Ethnicity and Nationalism. Stone, J., et al. (Eds.) UK:Wiley-Blackwell.

Benwell, M. 2017. "Argentine territorial nationalism in the South Atlantic and Antarctica". En Dodds, K., Hemmings, A., Roberts, P. (Eds.). 2017. Handbook on the Politics of Antarctica. Cheltenham: Edward Elgar. 540-554.

Benwell, M., Núñez, A., \& Amigo, C. 2018. "Flagging the nations: citizen's active engagements with everyday nationalism in Patagonia, Chile". Area.

Bhabha, H. 2010. Nación y narración. Entre la ilusión de una identidad y las diferencias culturales. Buenos Aires: Siglo Veintiuno Editores.

Bhabha, H. 2011. Nuevas minorías, nuevos derechos. Buenos Aires: Siglo Veintiuno Editores.

Billig, M. 1995. Banal Nationalism. London: SAGE.

Castoriadis, C. 2010. "El imaginario social y la institución. Lo histórico social". La institución imaginaria de la sociedad. Argentina: Ensayo. 269-351.

Carrasco, P. (2017). "Relatos Orales, Rastros de la Identidad de la Patagonia-Aysén". En Núñez, A., Aliste, E., Bello, A., y Osorio, M. (Eds.). 2017. Imaginarios geográficos, prácticas y discursos de frontera. Aisén-Patagonia desde el texto de la nación. Santiago: LOM. 191-212.

Collins, B., Coleman, T. 2008. "Social Geographies of education: looking within, and Beyond, school boudaries". London, UK, Geography Compass 2/1. 281-299.

Dardel, E. 2013. El hombre y la tierra: naturaleza de la realidad geográfica. Madrid: Biblioteca Nueva.

De Certeau, M. 2010. La invención de lo cotidiano. Artes de hacer. México D.F.: Iberoamericana.

Deleuze, G. 2006. Exasperación de la filosofía. El Leibniz de Deleuze. Buenos Aires: Cactus.

Deleuze, 2002. Nietzsche y la filosofía. Barcelona, Anagrama.

Dittmer, J. \& Dodds, K. 2008. Popular geopolitics past and future: Fandom, identities and audiences. Geopolitics. 13(3): 437-457.

Dirección de Vialidad Ministerio de Obras Públicas de Chile. 1982. Estudio de la Dirección de Vialidad del Ministerio de Obras Públicas. Santiago: MOP.

Focault, M. 2012. Vigilar y castigar: Nacimiento de la prisión. España: Biblioteca Nueva

Gellner, E. 1983. Nations and Nationalism. New York: Cornell University Press.

Ivanoff, D. 1996. La Guerra de Chile Chico o los Sucesos del Lago Buenos Aires. Valdivia: Ediciones Cerro Azul.

Ivanoff, D. 2013. Bolicheros y Pobladores. Una relación de frontera. Santiago: Lom Ediciones.

Jones, R., \& Merriman, P. 2009. "Hot, banal and everyday nationalism: Bilingual road signs in Wales". political Geography. 164-173.
Latour, B. 2008. Reensamblar lo social. Buenos Aires: Manantial.

Lussault, M. 2015. El hombre espacial. La construcción social del espaio humano. Buenos Aires, Amorrortu.

Manterola, L. 2009. Control Fronterizo Integrado Los Libertadores. Proyecto de Título - Repositorio Universidad de Chile. Disponible en:http://repositorio.uchile.cl/tesis/uchile/2009/ aq-manterola_l/pdfAmont/aq-manterola_I.pdf

Massey, D. 2012. "Un sentido global del lugar". Doreen Massey, sentido global del lugar. Albet, A. y Benach, N. (Eds.). Barcelona: Icaria. 112-129.

Ministerio de Obras Públicas (CHI) \& Ministerio de Planificación Federal, Inversión Pública y Servicios (ARG). 2012. Estudio binacional de conectividad Argentina - Chile. Banco Interamericano de Desarrollo. Disponible en: http:// www.dirplan.cl/centrodedocumentacion/Documents/Estudios/ Desarrollados/2012/conect_chile_arg/Conectividad_Argentina_ Chile_informe_final_agosto_2012.pdf. (consultado en abril de 2018)

Núñez, A. 2013. "Definiendo una geografía para la nación: resignificación territorial de Chile, siglos XVIII-XIX". En Bonastra, Q. y Jori, G. Imaginar, organizar y controlar el territorio. Una visión geográfica de la construcción del Estado-nación. Barcelona, Icaria Editorial. 167-196.

Núñez, A., Aliste, E. y Bello, A. 2014. "El discurso del desarrollo en Patagonia-Aysén: la conservación y la protección de la naturaleza como dispositivos de una renovada colonización". Scripta Nova 18 (493): 46

Núñez, 2011, "El aislamiento geográfico: un asunto de perspectivas". En Arenas, Salazar y Núñez, 2011. El aislamiento geográfico ¿Problema u oportunidad?. Santiago, Geolibros. 1523.

Núñez, A., Aliste, E., Bello, A., y Osorio, M. (Eds.). 2017. Imaginarios geográficos, prácticas y discursos de frontera. AisénPatagonia desde el texto de la nación. Santiago: LOM. 167-190.

Núñez, A., Baeza B., y Benwell, M. 2017. "Cuando la nación queda lejos: fronteras cotidianas en el paso Lago Verde (AysénChile) / Aldea Las Pampas (Chubut-Argentina)". Revista de Geografía Norte Grande. 66: 5-8.

Núñez, A., Aliste, E., Bello, A., \& Astaburuaga, J. 2019. Ecoextractivismo y los discursos de la naturaleza en Patagonia-Aysén: nuevos imaginarios geográficos y renovados procesos de control territorial. Revista Austral De Ciencias Sociales, (35), 133-153.

Oliveira Jr, W. 2009. "Grafar o espaço, educar os olhos. Rumo a geografias menores". Pro-Posições. 20(3): 17-28.

Said, E. 2010. Orientalismo. Barcelona, Editorial Debate.

Serje, Margarita. El revés de la nación. Territorios salvajes, fronteras y tierras de nadie. Bogotá: Ediciones Universidad de los Andes, 2005. 295 pgs.

Tuan, Yi-Fu. 1974. Topophilia. A study of environmental perception, attitudes and values. New Jersey: Prentice-Hall Inc.

Zusman, P. 2013. "La geografía histórica, la imaginación y los imaginarios geográficos". Revista de Geografía Norte Grande. 54: 51-66. 